\title{
Adaptive study design to assess effect of TRPV4 inhibition in patients with chronic cough
}

\author{
Valerie J. Ludbrook ${ }^{1}$, Kate E. Hanrott ${ }^{1}$, James L. Kreindler ${ }^{2}$, Joanna E. Marks-Konczalik ${ }^{1 \dagger}$, Nick P. Bird ${ }^{1}$, \\ Debbie A. Hewens ${ }^{1}$, Misba Beerahee ${ }^{1}$, David J. Behm², Alyn Morice $\mathbb{C}^{3}$, Lorcan McGarvey ${ }^{4}$, Sean M. Parker ${ }^{5}$, \\ Surinder S. Birring ${ }^{6}$ and Jaclyn Smith $\mathbb{D}^{7}$
}

${ }^{1}$ GlaxoSmithKline, Stevenage, UK. ${ }^{2}$ GlaxoSmithKline, Upper Providence, USA. ${ }^{3}$ Hull York Medical School, Cottingham, UK. ${ }^{4}$ Queens University, Belfast, UK. ${ }^{5}$ North Tyneside General Hospital, North Shields, UK. ${ }^{6}$ Centre for Human \& Applied Physiological Sciences, School of Basic \& Medical Biosciences, Faculty of Life Sciences \& Medicine, King's College London, London, UK. ${ }^{7}$ Division of Infection Immunity and Respiratory Medicine, University of Manchester and Manchester University NHS Foundation Trust, Manchester, UK.

Corresponding author: Valerie Ludbrook (Valerie.J.Ludbrook@GSK.com)

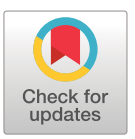

Copyright $\odot$ The authors 2021

This version is distributed under the terms of the Creative Commons Attribution NonCommercial Licence 4.0. For commercial reproduction rights and permissions contact permissions@ersnet.org

Received: 19 April 2021 Accepted: 4 June 2021

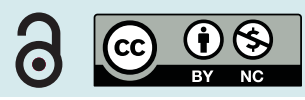

Shareable abstract (@ERSpublications)

Adaptive study design allowed the effect of TRPV4 inhibition on chronic cough to be explored and stopped for futility after only 17 participants. This approach could be used more to limit the exposure of participants to ineffective investigational drugs. https://bit.ly/3wdl4H9

Cite this article as: Ludbrook VJ, Hanrott KE, Kreindler JL, et al. Adaptive study design to assess effect of TRPV4 inhibition in patients with chronic cough. ERJ Open Res 2021; 7: 00269-2021 [DOI: 10.1183/ 23120541.00269-2021].

\section{Abstract}

Objective Airway sensory nerves involved in the cough reflex are activated by adenosine triphosphate (ATP) agonism of P2X purinoceptor 3 (P2X3) receptors. Transient receptor potential vanilloid 4 (TRPV4) channel activation causes ATP release from airway cells, and it is hypothesised that a TRPV4-ATP-P2X3 axis contributes to chronic cough. An adaptive study was run to determine if TRPV4 inhibition, using the selective TRPV4 channel blocker GSK2798745, was effective in reducing cough.

Methods A two-period randomised, double blinded, placebo-controlled crossover study was designed with interim analyses for futility and sample size adjustment. Refractory chronic cough patients received either GSK2798745 or placebo once daily for 7 days with a washout between treatments. Pharmacokinetic samples were collected for analysis of GSK2798745 at end of study. The primary end-point was total cough counts assessed objectively during day-time hours $(10 \mathrm{~h})$ following 7 days of dosing.

Results Interim analysis was performed after 12 participants completed both treatment periods. This showed a 32\% increase in cough counts on Day 7 for GSK2798745 compared to placebo; the pre-defined negative criteria for the study were met and the study was stopped. At this point 17 participants had been enrolled (mean 61 years; 88\% female), and 15 had completed the study. Final study results for posterior median cough counts showed a 34\% (90\% credible interval: $-3 \%$, +85\%) numerical increase for GSK2798745 compared to placebo.

Conclusion There was no evidence of an anti-tussive effect of GSK2798745. The study design allowed the decision on lack of efficacy to be made with minimal participant exposure to the investigational drug.

\section{Introduction}

Chronic cough is defined in clinical practice as cough lasting longer than 8 weeks [1], is highly prevalent worldwide and is a leading cause of unplanned visits to the doctor's office [2]. Regardless of aetiology, chronic cough is difficult to treat and can significantly diminish quality of life [3] and the ability to perform everyday activities. Despite recent advances in treating cough [4], there remains a significant unmet need for safe and effective medicines [5].

It has been previously hypothesised that heightened cough reflex sensitivity is mediated by adenosine triphosphate (ATP) through P2X purinoceptor 3 (P2X3) receptors [6, 7]. Transient receptor potential vanilloid 4 (TRPV4) is an ion channel broadly expressed in the respiratory tract and is activated by a wide range of stimuli including temperature, $\mathrm{pH}$ and osmolarity $[8,9]$. TRPV4 activation causes ATP release 
from airway epithelial cells [10], and studies have established a role for TRPV4-mediated ATP release and P2X3 receptor modulation in the activation of airway afferents [10]. Therefore, it is possible that a TRPV4-ATP-P2X3 axis contributes to the evolution and persistence of chronic cough. Moreover, clinical data suggest that pharmacological inhibition of P2X3 receptors reduces cough frequency and improves patient-reported outcomes and quality of life in a subset of patients with chronic cough [11-13].

GSK2798745 is a selective TRPV4 channel blocker, and the aim of this study was to evaluate whether blockade of TRPV4 channels is effective in reducing cough in patients with idiopathic or treatment refractory chronic cough.

\section{Methods}

\section{Study design and population}

This multicentre, placebo-controlled, double-blind, randomised, two-period crossover study was conducted from April until October 2018 in the UK. The study was approved by the appropriate ethics committee and was conducted in accordance with the International Council on Harmonisation of Technical Requirements for Registration of Pharmaceuticals for Human Use and Good Clinical Practice. All participants provided written informed consent (ClinicalTrials.gov identifier NCT03372603; EudraCT number 2017-002265-21).

The study design is detailed in figure 1 . An automated system was used to randomly assign participants who were stratified according to inclusion in a previous cough study within the last 12 months. The study design included up to two interim analyses to be conducted for futility and possible sample size adjustment. The protocol is published at www.gsk-studyregister.com/en/trial-details/?id=207702.

The targeted population were participants aged 18 to 75 years with treatment refractory or idiopathic chronic cough for $\geqslant 1$ year that was unresponsive to targeted treatment or for which no identifiable cause was determined. Additional inclusion criteria included a score of $\geqslant 40 \mathrm{~mm}$ on the Cough Severity Visual Analogue Scale (VAS) at screening, no significant findings on chest imaging within 12 months before screening, a forced expiratory volume in $1 \mathrm{~s}\left(\mathrm{FEV}_{1}\right) \geqslant 80 \%$ of the predicted normal value at screening, or documented evidence of $\mathrm{FEV}_{1} \geqslant 80 \%$ within the 6 months before screening, and a body weight $>50 \mathrm{~kg}$ and body mass index within the range of 18 to $40 \mathrm{~kg} \cdot \mathrm{m}^{-2}$.

Key exclusion criteria were current smoker, history of smoking or a cumulative smoking history of $\geqslant 20$ years; history or current evidence of any serious or clinically significant condition; history or current evidence of chronic productive cough; and respiratory infection within 6 weeks of screening.

\section{Study methods}

The primary objective of the study was to compare the efficacy of 7 days oral dosing of GSK2798745 tablets with placebo tablets. The primary end-point for the study was total cough counts during day-time hours (total number of coughs during the first $10 \mathrm{~h}$ following dosing - during which time participants were instructed to be awake) following 7 days of dosing. Coughs were recorded for $24 \mathrm{~h}$ prior to dosing of each

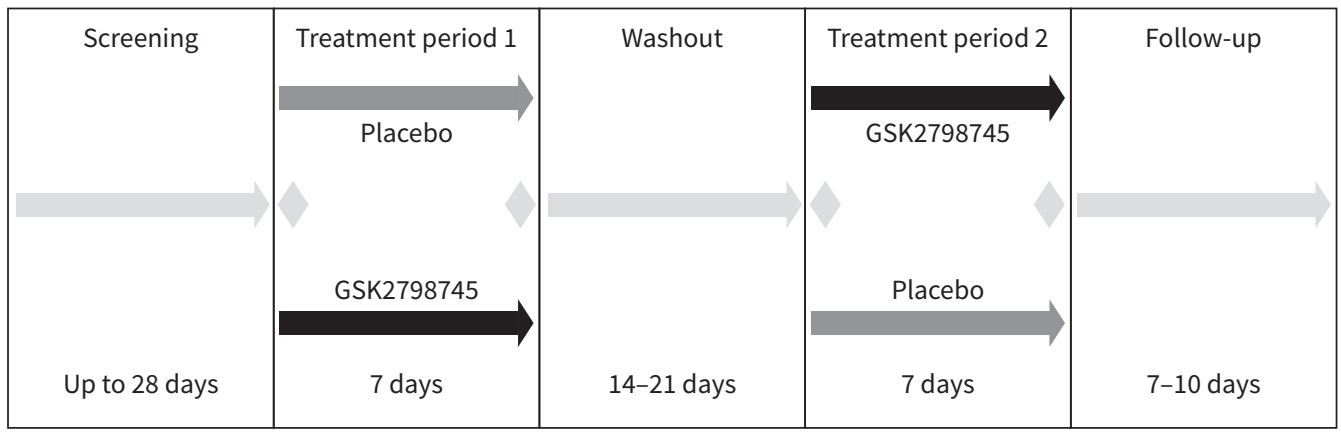

24-h cough recording at the beginning and end of each treatment period

FIGURE 1 Study design. Participants were randomised to receive either $4.8 \mathrm{mg}$ GSK2798745 on Day 1, followed by $2.4 \mathrm{mg}$ once daily for 6 days, or matching placebo once daily for 7 days in the first treatment period, followed by the alternative regimen in the second treatment period. 
treatment period (baseline) and for $24 \mathrm{~h}$ following dosing on Day 7 of each treatment period. Both day-time and 24-h cough counts were assessed.

Objective assessment of cough was done using the VitaloJAK cough recording device [14]. The Leicester Cough Questionnaire (LCQ) was utilised as a quality-of-life measure of chronic cough [15]. In addition, participants were asked to complete two VAS forms, one each to rate the severity of their cough and their urge to cough.

Evaluation of safety was based on reported adverse events, clinical laboratory values, vital signs and electrocardiogram (ECG).

Plasma samples collected on Day 1 and Day 7: predose, 0.25, 0.5, 1, 1.5, 2, 2.5, 3, 3.5 h post dose; and on Day 8: $24 \mathrm{~h}$ post dose were analysed for GSK2798745. A validated analytical method was used, based on solid phase extraction, followed by HPLC-MS/MS analysis [16]. The lower limit of quantification was $0.2 \mathrm{ng} \cdot \mathrm{mL}^{-1}$ using a $100-\mu \mathrm{L}$ aliquot of human plasma with a higher limit of quantification of $100 \mathrm{ng} \cdot \mathrm{mL}^{-1}$. For each analytical method, quality control samples containing three different analyte concentrations were analysed with each batch of samples against separately prepared calibration standards. For the analysis to be acceptable, no more than one-third of the total quality control results and no more than one-half of the results from each concentration level were to deviate from the nominal concentration by $>15 \%$. The applicable analytical runs met all pre-defined run acceptance criteria.

\section{Analyses}

The target number of evaluable participants was 24. A sample size re-estimation was planned during the study, allowing a maximum of 48 participants to be enrolled.

Day-time 10-h cough counts were analysed by first log transforming the counts recorded on Day 7 of each dosing period, hence the treatment effect was presented as a ratio GSK2798745/placebo. The statistical analysis was based on a Bayesian mixed effects model assuming a non-informative prior distribution. Treatment and period were included as fixed effects and terms related to baseline cough counts were included in the model as covariates. The presence of carryover effects or treatment by period interaction was also investigated. In addition, effects pertaining to the centre and participation in a cough-related clinical trial in the previous 12 months were investigated. The posterior probability and corresponding $90 \%$ credible intervals that the true ratio of the mean effect size of the test treatment and the mean effect size of the placebo treatment, $\mu$ (test) $/ \mu$ (placebo), was $<0.7$ constructed, i.e. a day-time cough count total of at least $30 \%$ less for GSK2798745 than for placebo was of interest. In addition, the posterior probability true effect size distribution was used to obtain estimates for the probabilities that the true effect size falls below 1 and 0.5 , representing any reduction and a 50\% reduction in day-time cough count totals for GSK2798745 compared with placebo.

Prior to analysis, data were assessed for any missing intervals of cough counts during the $10 \mathrm{~h}$ period, e.g. if the participant removed the device temporarily. Missing data were pro-rated if at least $60 \%$ of the first $10 \mathrm{~h}$ of monitoring included valid cough monitoring data, i.e. the monitor was attached and working for at least 6 out of the first $10 \mathrm{~h}$. For example, if a participant recorded 200 coughs between 0 and $8 \mathrm{~h}$ but the next $2 \mathrm{~h}$ were not recorded, the missing $2 \mathrm{~h}$ would be estimated as 50, resulting in an imputed $10 \mathrm{~h}$ total of 250 coughs. Similar rules were applied to the 24-h data.

\section{Interim analysis}

One interim analysis was conducted during the study when 12 participants completed both dosing periods. A key objective of the interim analysis was to test for futility, and with a sample size of 12 participants, there was a high probability of being able to stop the study early and reduce patient exposure if the drug was truly placebo-like and, similarly, a low probability of stopping for futility if the drug was truly effective. The treatment level results were made available to the GSK study team who reviewed the available cough count data before deciding on whether to stop the study on the grounds of futility, or adjust the sample size.

To assess futility, the results from the interim analysis were compared with pre-defined criteria for a negative study; to be declared negative, a posterior probability of $<30 \%$ that the true treatment ratio (GSK2798745/placebo) was $<0.7$, i.e. posterior probability (ratio $<0.7$ ) $<30 \%$, was required.

The interim analysis included a preliminary assessment of whether there was any evidence for the presence of carryover effects or treatment by period interaction. 
Only the responsible GSK statisticians and programmers had access to individual participant data. However, the findings of the interim analysis were shared with the entire GSK study team.

Results

Study population

A total of 34 participants were screened, and 17 (50\%) failed screening, primarily for not meeting the inclusion/exclusion criteria (figure 2). An interim analysis was conducted after 12 participants had completed both dosing periods, and the study was terminated on grounds of futility. Recruitment was not halted during the interim analysis; therefore, a total of 17 participants were randomised into the study. Dosing of two participants, whose dosing was ongoing at the time the study was terminated, was stopped, so only 15 of the 17 participants completed the study as planned, and only 16 of those had been dosed with GSK2798745.

Baseline characteristics of the population are presented in table 1.

\section{Efficacy}

Statistical analysis at the interim $(\mathrm{n}=12)$ showed a 32\% increase in day-time cough counts on Day 7 for GSK2798745 compared with placebo. The pre-defined negative criteria for the study were met and the study was subsequently stopped. Final study $(n=17)$ statistical analysis results for both day-time and $24-\mathrm{h}$ cough counts are shown in tables 2 and 3. Individual participant change from baseline for day-time and 24-h cough counts are presented in figure 3.

Severity of Cough and Urge to Cough VAS scores and LCQ results (both individual domains and total) did not demonstrate a positive effect of GSK2798745 on cough as presented in figure 4.
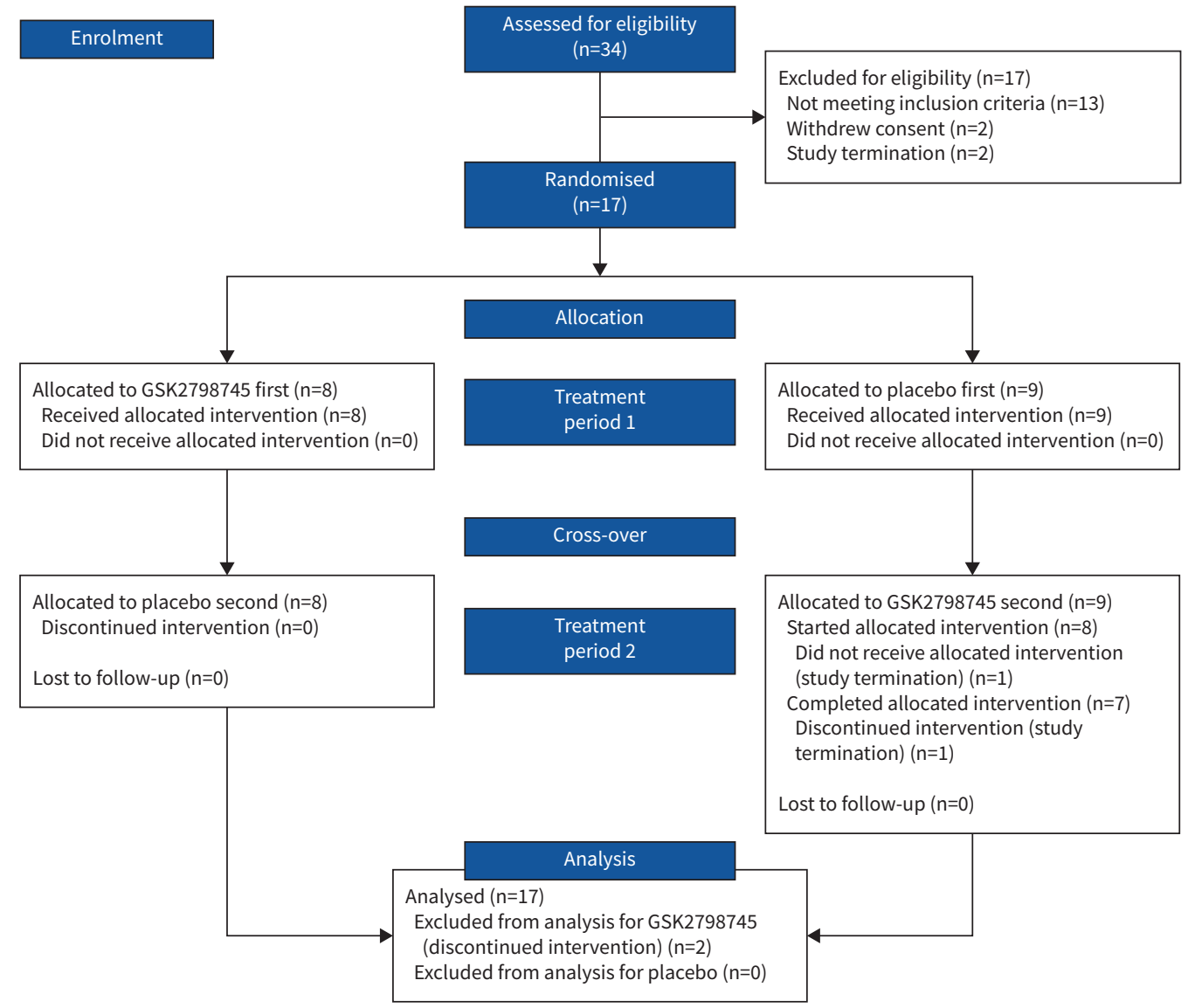

FIGURE 2 Consolidated Standards of Reporting Trials participant disposition. 


\begin{tabular}{|c|c|c|}
\hline Demographics & \multicolumn{2}{|c|}{ Total } \\
\hline Age years ${ }^{\#}($ mean $\pm s \mathrm{D})$ & \multicolumn{2}{|c|}{$61.0 \pm 9.85$} \\
\hline \multicolumn{3}{|l|}{ Age range years ${ }^{\#}$} \\
\hline $18-64$ & \multicolumn{2}{|c|}{$10(59)$} \\
\hline $65-74$ & \multicolumn{2}{|c|}{$7(41)$} \\
\hline \multicolumn{3}{|l|}{ Sex } \\
\hline Female & \multicolumn{2}{|c|}{$15(88)$} \\
\hline Male & \multicolumn{2}{|c|}{$2(12)$} \\
\hline BMI $\mathrm{kg} \cdot \mathrm{m}^{-2}(\mathrm{mean} \pm \mathrm{SD})$ & \multicolumn{2}{|c|}{$27.61 \pm 4.96$} \\
\hline Height $(\mathrm{cm})($ mean $\pm \mathrm{sD})$ & \multicolumn{2}{|c|}{$162.9 \pm 6.58$} \\
\hline Weight (kg) (mean \pm sD) & \multicolumn{2}{|c|}{$73.19 \pm 13.3$} \\
\hline \multicolumn{3}{|l|}{ Ethnicity } \\
\hline Hispanic or Latino & \multicolumn{2}{|c|}{0} \\
\hline Not Hispanic or Latino & \multicolumn{2}{|c|}{$17(100)$} \\
\hline \multicolumn{3}{|l|}{ Race } \\
\hline Asian - South East Asian Heritage & \multicolumn{2}{|c|}{$1(6)$} \\
\hline \multirow{2}{*}{ White - White/Caucasian/European Heritage } & \multicolumn{2}{|c|}{$16(94)$} \\
\hline & GSK2798745 & Placebo \\
\hline Baseline day-time $10-\mathrm{h}$ cough count (geometric mean $(95 \% \mathrm{CI})$ ) & $205.2(116.7-360.6)$ & $242.5(144.7-406.4)$ \\
\hline Baseline Cough Severity VAS (mean \pm SD) & $53.9 \pm 18.5$ & $57.2 \pm 23.35$ \\
\hline Baseline Urge to Cough VAS (mean $\pm \mathrm{sD}$ ) & $58.6 \pm 18.4$ & $56.2 \pm 26.81$ \\
\hline Baseline LCQ (mean \pm SD) & $12.07 \pm 3.6$ & $11.62 \pm 3.49$ \\
\hline
\end{tabular}

Safety and pharmacokinetics

There were no serious adverse events, deaths or other significant adverse events reported during this study. A summary of adverse events is presented in table 4.

There were no clinically significant changes in clinical laboratory assessments, ECGs and vital signs (systolic and diastolic blood pressure, heart rate, temperature and weight), and no changes in ECGs and vital signs were reported as adverse events.

GSK2798745 plasma concentrations achieved at peak and trough were within the expected range. Data are summarised in table 5.

\section{Discussion}

This is the first study to evaluate the effect of TRPV4 antagonism in patients with refractory chronic cough. Treatment with the TRPV4 antagonist GSK2798745, however, did not result in any anti-tussive effect when compared with placebo. The quality of study data was considered high, with a minimal amount of missing cough count data. The adaptive design of the study allowed for an interim analysis after 12 participants had completed.

\section{TABLE 2 Statistical analysis model adjusted cough count estimates}

\begin{tabular}{lcccc} 
Treatment & $\mathbf{N}$ & $\mathrm{n}$ & Posterior median \pm SD & 95\% credible interval \\
\hline $\begin{array}{l}\text { Day-time } \\
\text { 10-h total cough counts treatment estimates }\end{array}$ & & \\
Placebo & 17 & 17 & $180.57 \pm 24.76$ & $(137.85-235.45)$ \\
GSK2798745 & 16 & 15 & $241.11 \pm 35.41$ & $(181.38-320.60)$ \\
24-h total cough counts treatment estimates & 17 & $413.44 \pm 43.56$ & $(336.35-508.34)$ \\
Placebo & 17 & 15 & $450.68 \pm 50.8$ & $(361.50-561.70)$ \\
\hline
\end{tabular}

Total cough counts were log-transformed prior to analysis. $\mathrm{N}=$ number of participants in analysis population; $\mathrm{n}=$ number of participants providing data. 


\section{TABLE 3 Statistical analysis treatment comparisons}

Treatment comparison Posterior median ratio $\pm \mathrm{SD}$

$90 \%$ credible interval

Posterior prob. of true Diff

$<1.0<0.7 \quad<0.5$

Day-time 10-h total cough counts treatment comparison

GSK2798745/Placebo $\quad 1.34 \pm 0.27$

24-h total cough counts treatment comparison

GSK2798745/Placebo $\quad 1.09 \pm 0.17$

Note a sensitivity analysis using non-imputed data: $1.31(0.95-1.8090 \%$ credible interval) for $10 \mathrm{~h}$ and 1.09 (0.85-1.40) for $24 \mathrm{~h}$.

Day-time cough counts

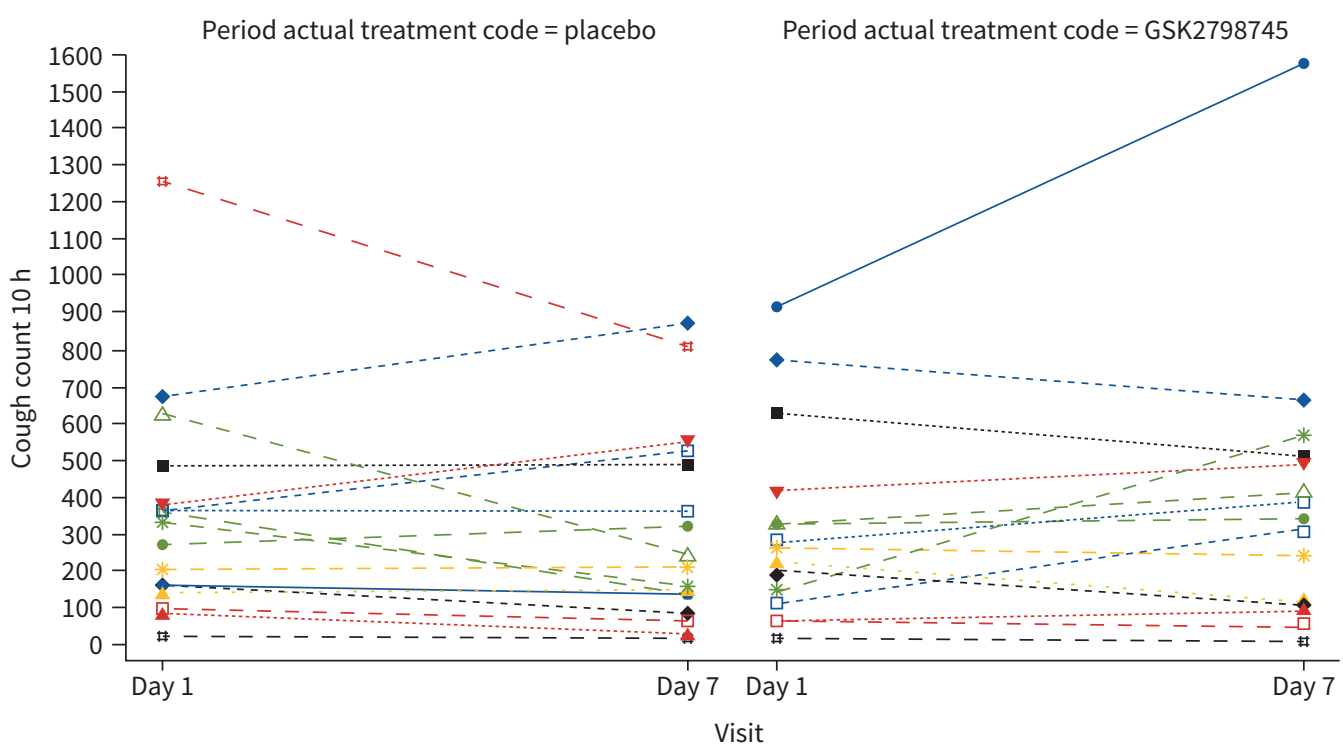

24-h cough counts

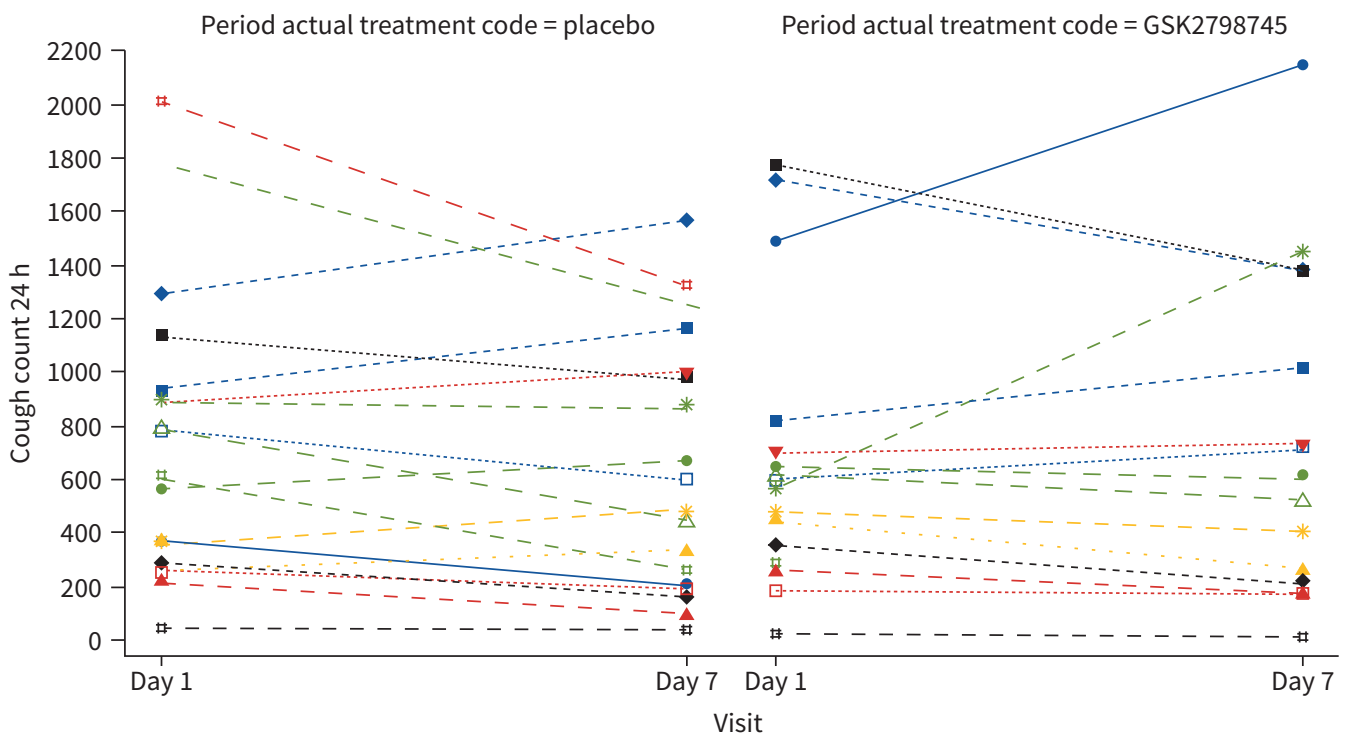

FIGURE 3 Individual change from baseline cough counts over day-time and 24-h time periods. Individual patient data are plotted on each line. 

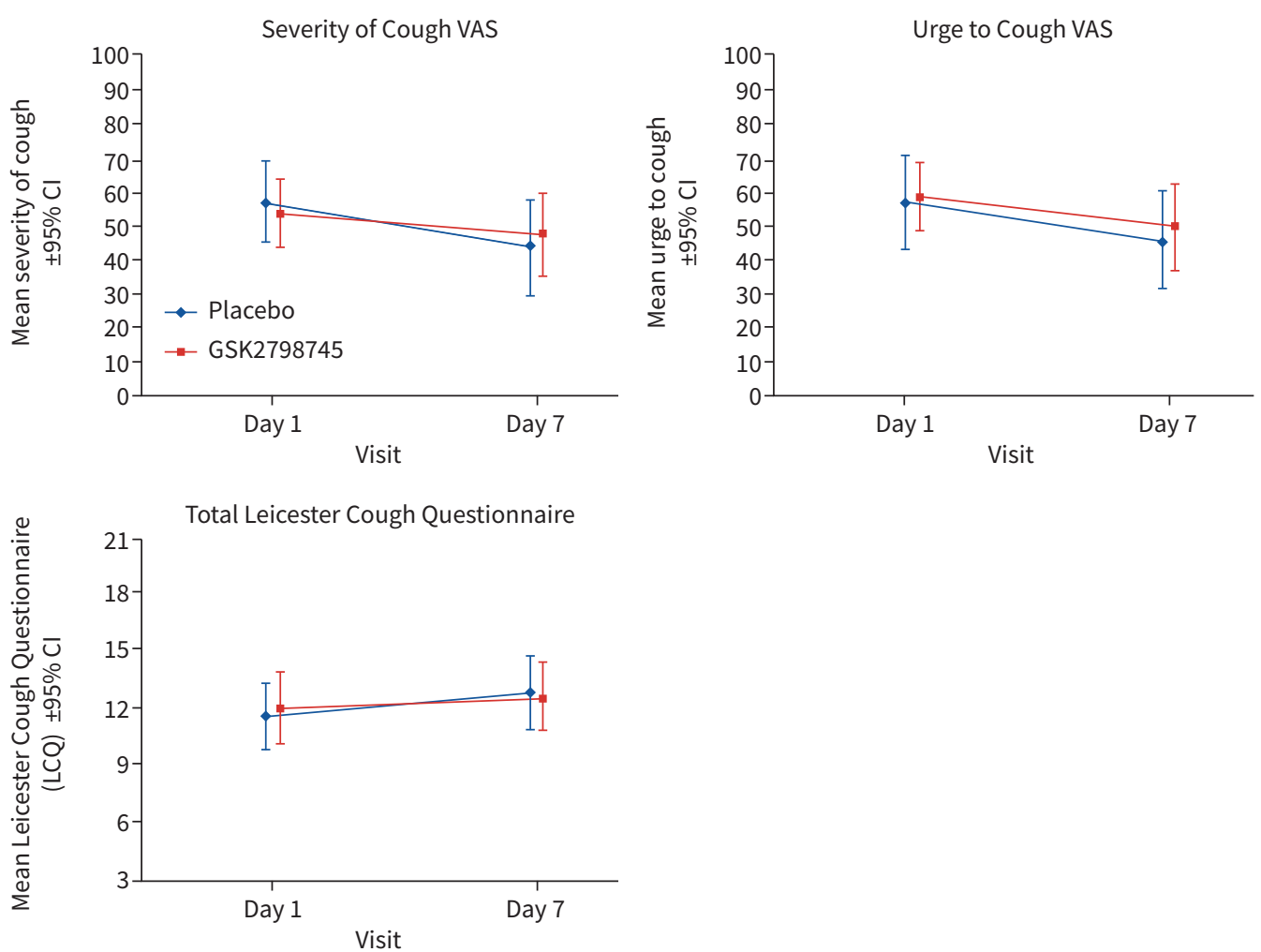

FIGURE 4 Patient reported outcome measure results for Severity of Cough visual analogue scale (VAS), Urge to Cough VAS and Leicester Cough Questionnaire (LCQ), presented as mean $\pm 95 \% \mathrm{Cl}$.

The results from the interim analysis demonstrated that there was no clear difference in Day 7 day-time cough counts between the two treatment groups, and no carryover effect or treatment by period interaction was observed; therefore, the results met the negative study criteria, and the study was terminated early. Final data analysis included 17 enrolled participants with 15 completers, and the result was consistent with the interim analysis. The fact that increasing the number of participants treated did not affect the outcome of the results supports the use of this type of study design to minimise the exposure of patients to ineffective investigative treatments in studies. In adaptive design clinical studies, it is considered important to establish statistically based decision rules prior to starting the study $[17,18]$. The results at the interim demonstrated that the posterior probability that the true ratio of the mean effect size was $<0.7$ was just $1.0 \%$, thereby clearly meeting the pre-defined criteria for stopping for futility. This decision prevented the target patient recruitment of 24 participants being exposed to an ineffective treatment.

\section{TABLE 4 Most frequent adverse events observed in the study}

\begin{tabular}{lccc} 
& Placebo & GSK2798745 & Total \\
\hline Subjects $n$ & 17 & 16 & 17 \\
Number of participants with any event & $9(53)$ & $11(69)$ & $13(76)$ \\
\hline Number of participants with a drug-related event & $2(12)$ & $2(13)$ & $4(24)$ \\
Most common adverse events: (adverse events occurring in & 2 participants in any treatment group) & \\
Headache & $3(18)$ & $7(44)$ & $8(47)$ \\
Diarrhoea & $2(12)$ & $1(6)$ & $3(18)$ \\
Fatigue & $1(6)$ & $2(13)$ & $3(18)$ \\
\hline Oropharyngeal pain & $2(12)$ & 0 & $2(12)$ \\
\hline
\end{tabular}

Data expressed as $\mathrm{n}(\%)$ unless otherwise noted. Note: Adverse events were assigned to a treatment group based on the start date of the adverse events in relation to the study treatment period. Adverse events that started during the washout period were assigned to the Period 1 treatment group. 


\begin{tabular}{|c|c|c|c|c|c|c|}
\hline Parameter & Visit $^{\#}$ & $\mathrm{~N}$ & $\mathrm{n}$ & Geometric mean & 95\% Cl (lower, upper) & $\% \mathrm{CVb}^{\bullet}$ \\
\hline \multirow[t]{2}{*}{$\mathrm{C}_{\max } \mathrm{ng} \cdot \mathrm{mL}^{-1}$} & Day 1 & 16 & 16 & 17.862 & (14.468-22.052) & 41.1 \\
\hline & Day 7 & & 15 & 10.959 & $(9.425-12.741)$ & 27.7 \\
\hline \multirow[t]{2}{*}{$\operatorname{AUC}(0-3.5) \mathrm{h} \cdot \mathrm{ng} \cdot \mathrm{mL}^{-1}$} & Day 1 & 16 & 16 & 36.156 & (28.328-46.148) & 48.3 \\
\hline & Day 7 & & 15 & 26.151 & (22.479-30.424) & 27.8 \\
\hline $\mathrm{C}_{24} \mathrm{ng} \cdot \mathrm{mL}^{-1}$ & Day 7 & 16 & 15 & 2.4179 & $(1.7295-3.3803)$ & 66.5 \\
\hline \multirow[t]{2}{*}{$T_{\max } \mathrm{h}^{+}$} & Day 1 & 16 & 16 & 2.500 & $(1.00-3.50)$ & NA \\
\hline & Day 7 & & 15 & 2.500 & $(1.00-3.50)$ & NA \\
\hline
\end{tabular}

AUC: area under the curve. \#: day 1 dose $4.8 \mathrm{mg}$; Days 2-7 dose $2.4 \mathrm{mg}$. ": coefficient of variability between participants. ${ }^{+}: T_{\max }$ expressed as median and range.

The loading dose of $4.8 \mathrm{mg}$ followed by repeat doses of $2.4 \mathrm{mg}$ once daily was chosen to provide adequate systemic drug levels for TRPV4 receptor target engagement while being within the exposure limits pre-defined by pre-clinical toxicology data. This dose regimen showed dose proportional change in systemic exposure to drug, albeit based on the limited sampling scheme. The GSK2798745 drug levels achieved at peak and trough are within the expected range for pharmacological inhibition of TRPV4 based on an estimated $\mathrm{IC}_{50}$ of 2.1-3.2 $\mathrm{ng} \cdot \mathrm{mL}^{-1}$ for GSK2798745 obtained from in vivo pre-clinical and ex vivo clinical pharmacokinetics-pharmacodynamics (PK-PD). The steady-state average peak ( $\mathrm{C}_{\max }$ $\left.10.9 \mathrm{ng} \cdot \mathrm{mL}^{-1}\right)$ and trough $\left(\mathrm{C}_{24} 2.4 \mathrm{ng} \cdot \mathrm{mL}^{-1}\right)$ concentrations of GSK2798745 equate to $\sim 80 \%$ and $\sim 50 \%$ inhibition of TRPV4 receptors, respectively. The levels of predicted TRPV4 inhibition obtained in this study could be considered adequate for modulating cough considering the level of target occupation necessary for therapeutic effect is typically $\sim 60-90 \%$ for antagonists of multiple drug targets, including ion channels [19]. However, caution should be taken when interpreting the PK-PD predictions from this study, because the in vivo and ex vivo PK-PD models were based on the vascular endothelial cell as the site of pharmacological action; GSK2798745 levels might differ in the airways, the potential TRPV4 site of action for modulating cough [10].

TRPV4 is one of several ion channels to have been implicated in the cough reflex [20, 21], and there has been much interest in the TRP receptor family as therapeutic targets in cough [22, 23]. There is significant pre-clinical evidence indicating involvement of TRPV1, TRPA1 and now TRPV4 in the cough reflex; however, so far, none have shown translation of this to efficacy in human clinical studies when performed [23-25]. There may be several reasons for this. Firstly, it has become clear in recent years that while evoked cough models are important for elucidating mechanisms and confirming target engagement, in both animals and humans, they are poorly predictive of impact on pathological cough in a real-life clinical population [22]. This may be as a result of redundancy in the system, or it is possible that inhibition of one of the TRP receptors results in the induction of other compensatory mechanisms. Secondly, only for TRPV1 antagonists have negative findings been reported in the presence of positive effects on capsaicin challenge confirming target engagement in the lung; without this there is always the possibility of inadequate dosing/potency/receptor occupancy [25]. In this study, it could also be that the dose was not high enough for the reasons described above. For TRPV4 there is evidence from the pre-clinical studies (using a TRPV4 agonist and hypotonic solutions) that it is involved in activating afferent nerves causing cough through the release of ATP and activation of P2X3 [10], and there are data showing P2X3 inhibitors have reduced cough counts in patient studies $[11,12,13,26]$ demonstrating the importance of ATP and $\mathrm{P} 2 \mathrm{X} 3$ in the cough reflex.

Hypotonic cough challenges were not included in this study; therefore, evidence for TRPV4 target engagement in the lungs is lacking. However, unlike capsaicin and citric acid, hypotonic solutions are poor at evoking cough in both humans and animals. Methods used in the published literature are highly variable and established challenge agents, equipment and protocols suitable for use in multicentre clinical trials are lacking. More importantly, in the only published pre-clinical study using osmotic stimulation to evoke coughing, inhaled distilled water failed to demonstrate lung target engagement for a TRPV4 antagonist, questioning the value of this particular challenge [27]. In contrast, a recent study in humans showed that distilled water cough is significantly inhibited by a P2X3 antagonist [28], suggesting that additional triggers of P2X3/ATP-mediated cough could be contributing to the lack of efficacy seen with our TRPV4 inhibitor. So, while TRPV4 has been shown to be important in TRPV4 agonist activation of the TRPV4ATP-P2X3 axis in pre-clinical cough models, the evidence for it being important in chronic cough clinically is still not available. 
In conclusion, this study demonstrated that the level of TRPV4 inhibition achieved in this study using GSK2798745 was not effective in reducing cough in participants with idiopathic or treatment refractory chronic cough. The inclusion of a hypotonic cough challenge might have provided additional insights into whether TRPV4 target engagement in the lungs occurred, but the optimal challenge agent/protocol to assess this remains unclear. Using an adaptive study design allowed the study to be stopped for futility after only 17 participants had been enrolled. This approach could be used more to limit the exposure of chronic cough patients to ineffective investigational drugs.

Submitted article, peer reviewed.

Acknowledgements: The authors would like to thank the participants of the study as well as all the study staff for their contributions to the study. After completion of this study, sadly, Joanna Marks-Konczalik died. She significantly contributed to the study, and interpretation and discussion of results, prior to her death.

This study is registered at www.clinicaltrials.gov with identifier number NCT03372603 and at www. clinicaltrialsregister.eu with identifier number 2017-002265-21. Data availability: Individual deidentified data will not be shared. The protocol and statistical analysis plan are both published www.gsk-studyregister.com/en/ trial-details/?id=207702.

Conflict of interest: V.J. Ludbrook reports salary from and shares in GlaxoSmithKline, and part-funding of the present study from US Federal Government funds from the Department of Health and Human Services (Office of the Assistant Secretary for Preparedness and Response; Biomedical Advanced Research and Development Authority). K.E. Hanrott is an employee of and shareholder in GlaxoSmithKline. J.L. Kreindler is a full-time employee of and stockholder in AstraZeneca, and was a full-time employee of GlaxoSmithKline during the conduct of the study. N.P. Bird has nothing to disclose. D.A. Hewens is an employee of and shareholder in GlaxoSmithKline. M. Beerahee has nothing to disclose. D.J. Behm reports this project has been funded in part with Federal funds from the Department of Health and Human Services (Office of the Assistant Secretary for Preparedness and Response; Biomedical Advanced Research and Development Authority) under contract number HHSO100201700009C; and salary and bonuses from, and stock in GlaxoSmithKline. A. Morice reports grants from GSK during the conduct of the study; and grants and personal fees from Merck, Shionogi, Bayer, Bellus and NeRRi outside the submitted work. L. McGarvey reports personal fees from GSK, grants and personal fees from MERCK, personal fees from Shionogi, Bayer, Bellus Health and Nocion, grants and personal fees from Chiesi, and personal fees from Applied Clinical Intelligence, outside the submitted work. S.M. Parker has nothing to disclose. S.S. Birring reports personal fees from GSK, Merck, Bellus, Bayer, Shionogi, Nerre, Nocion and Boehringer Ingelheim, and grants from Merck, outside the submitted work. J. Smith reports grants from GlaxoSmithKline during the conduct of the study; grants and personal fees from NeRRe Pharmaceuticals, Menlo and Bayer, personal fees from Boehringer Ingleheim, nonfinancial support from Vitalograph, personal fees from Cheisi and Bellus, grants and personal fees from Axalbion, personal fees from AstraZeneca, grants and personal fees from Merck, and personal fees from Algernon and Nocion, outside the submitted work. In addition, J. Smith has a patent (A method for generating output data) licensed.

Support statement: This study has been funded by GlaxoSmithKline. This project has also been funded in part with US Federal Government funds from the Department of Health and Human Services (Office of the Assistant Secretary for Preparedness and Response; Biomedical Advanced Research and Development Authority) under Contract No. HHSO100201700009C. Funding information for this article has been deposited with the Crossref Funder Registry.

\section{References}

1 Irwin RS, French $\mathrm{CL}$, Chang $\mathrm{AB}$, et al. Classification of cough as a symptom in adults and management algorithms: CHEST guideline and expert panel report. Chest 2018; 153: 196-209.

2 Schappert SM, Rechtsteiner EA. Ambulatory medical care utilisation estimates for 2007. Vital Health Stat 2011; 13: 1-38.

3 Chamberlain SAF, Garrold R, Douiri A, et al. The impact of chronic cough: a cross-sectional European survey. Lung 2015; 193: 401-408.

4 Smith JA, Badri H. Cough: new pharmacology. J Allergy Clin Immunol Pract 2019; 7: 1731-1738.

5 Koskela HO, Latti AM, Purokivi MK. Long-term prognosis of chronic cough: a prospective, observational cohort study. BMC Pulm Med 2017; 17: 146.

6 Basoglu OK, Pelleg A, Essilfie-Quaye S, et al. Effects of aerosolized adenosine 5'-Triphosphate vs adenosine 5'-monophosphate on dyspnea and airway caliber in healthy nonsmokers and patients with asthma. Chest 2005; 128: 1905-1909. 
7 Ford AP, Undem BJ. The therapeutic promise of ATP antagonism at P2X3 receptors in respiratory and urological disorders. Front Cell Neurosci 2013; 7: 267.

8 Baxter M, Eltom S, Dekkak B, et al. Role of transient receptor potential and pannexin channels in cigarette smoke-triggered ATP release in the lung. Thorax 2014; 69: 1080-1089.

9 Toft-Bertelsen TL, Krízaj D, MacAulay N. When size matters: transient receptor potential vanilloid 4 channel as a volume-sensor rather than an osmo-sensor. J Physiol 2017; 595: 3287-3302.

10 Bonvini SJ, Birrell MA, Grace MS, et al. Transient receptor potential cation channel, subfamily V, member 4 and airway sensory afferent activation: role of adenosine triphosphate. J Allergy Clin Immunol 2016; 138: 249-261.

11 Abdulqawi R, Dockry R, Holt K, et al. P2X3 receptor antagonist (AF-219) in refractory chronic cough: a randomised, double-blind, placebo-controlled phase 2 study. Lancet 2015; 385: 1198-1205.

12 Smith J, Kitt M, Morice A, et al. Inhibition of P2X3 by MK-7264 reduces 24-hour cough frequency in a randomized, controlled, Phase 2b clinical trial. Eur Respir J 2017; 50: Suppl. 61, OA2932.

13 Muccino D, Green S. Update on the clinical development of gefapixant, a P2X3 receptor antagonist for the treatment of refractory chronic cough. Pulm Pharmacol Ther 2019; 56: 75-78.

14 McGuinness K, Holt K, Dockry SJ. Validation of the VitaloJAK 24 hour ambulatory cough monitor. Thorax 2012; 67: Suppl. 2, A131.

15 Birring S, Prudon B, Carr AJ, et al. Development of a symptom specific health status measure for patients with chronic cough: Leicester Cough Questionnaire (LCQ). Thorax 2003; 58: 339-345.

16 Goyal N, Skrdla P, Schroyer R, et al. Clinical pharmacokinetics, safety, and tolerability of a novel, first-in-class TRPV4 ion channel inhibitor, GSK2798745, in healthy and heart failure subjects. Am J Cardiovasc Drugs 2019; 19: $335-342$

17 Thorlund K, Haggstrom J, Park JJH, et al. Key design considerations for adaptive clinical trials: a primer for clinicians. BMJ 2018; 360: K698.

18 Pallmann P, Bedding AW, Choodari-Oskooei B, et al. Adaptive designs in clinical trials: why use them, and how to run and report them. BMC Medicine 2018; 16: 1.

19 Grimwood S, Hartig PR. Target site occupancy: emerging generalizations from clinical and preclinical studies. Pharmacol Ther 2009; 122: 281-301.

20 Nilius B. TRP channels in disease. Biochim Biophys Acta 2007; 1772: 805-812.

21 Grace MS, Dunuis E, Birrell MA, et al. TRP channel antagonists as potential antitussives. Chest 2012; 190: 11-15.

22 Bonvini SJ, Birrell MA, Smith JA, et al. Targetting TRP channels for chronic cough: from bench to bedside. Naunyn Schmiedebergs Arch Pharmacol 2015; 388: 401-420.

23 Roe NA, Lundy FT, Litherland GJ, et al. Therapeutic targets for the treatment of chronic cough. Curr Otorhinolaryngol Rep 2019; 7: 116-128.

24 Belvisi MG, Birrell MA, Wortley MA, et al. XEN-D0501, a novel transient receptor potential vanilloid1 antagonist does not reduce cough in patients with refractory cough. Am J Respir Crit Care Med 2017; 196: 1255-1263.

25 Khalid S, Murdoch R, Newlands A, et al. Transient receptor potential vanilloid 1 (TRPV1) antagonism in patients with refractory chronic cough: a double-blind randomized controlled trial. J Allergy Clin Immunol 2014; 134: 56-62.

26 Niimi A, Ishihara $\mathrm{H}$, Hida $\mathrm{H}$, et al. Phase 2a randomised, double-blind, placebo-controlled, crossover study of a novel P2X3 receptor antagonist S-600918 in patients with refractory chronic cough. Eur Respir J 2019; 54: Suppl. 63, RCT452.

27 Buday T, Kovacikova L, Ruzinak R, et al. TRPV4 antagonist GSK2193874 does not modulate cough response to osmotic stimuli. Respir Physiol Neurobiol 2017; 236: 1-4.

28 Morice AH, Kitt MM, Ford AP, et al. The effect of gefapixant, a P2X3 antagonist, on cough reflex sensitivity: a randomised placebo-controlled study. Eur Respir J 2019; 54: 1900439. 